\title{
Effect of Somatostatin-induced Suppression
}

\section{of Postprandial Insulin Response}

\section{upon the Hypertriglyceridemia}

\section{Associated with a High Carbohydrate Diet}

\author{
Henry N. Ginsberg, Alice Jacobs, Ngoc-Anh Le, and Jeffrey Sandler, \\ Department of Medicine, University of California at San Diego, \\ La Jolla, California 92093
}

A B S T R A C T In an attempt to define the relationship between plasma insulin and triglyceride concentrations, we have studied the effect of suppression of the postprandial insulin response upon the secretion and plasma concentration of very low density lipoprotein (VLDL)-triglycerides. Eight nondiabetic subjects with a wide range of fasting plasma triglyceride levels (100$358 \mathrm{mg} / \mathrm{dl}$ ) were studied during three dietary periods: base line, high carbohydrate ( $80 \%$ calories), and high carbohydrate ( $80 \%$ calories) with a daily intravenous infusion of somatostatin (SRIF) $(1.3 \mu \mathrm{g} / \mathrm{min})$ between 800 and $2,100 \mathrm{~h}$. The significant increase in postprandial insulin response observed during high carbohydrate vs. base line was completely abolished during high carbohydrate-SRIF. However, plasma triglyceride levels rose in all subjects during each high carbohydrate period (with/without SRIF) vs. base line and the mean values reached during each period were the same $(476 \pm 165$ vs. $482 \pm 152 \mathrm{mg} / \mathrm{dl}$, respectively). The secretion of VLDL-triglyceride into plasma was higher in four subjects, the same in two subjects, and lower in one subject during high carbohydrate-SRIF vs. high carbohydrate alone. The mean production rate of VLDL-triglyceride $(\mathrm{mg} / \mathrm{kg}$ per $\mathrm{h})$ was $25.6 \pm 4.9$ during the high carbohydrate and $40.9 \pm 28.1$ during

This work was presented in part in abstract form at the 39th Annual Meeting of the American Diabetes Association, June, 1979.

Reprint requests should be addressed to Dr. Ginsberg. Dr. Ginsberg's present address is Department of Medicine, The Mount Sinai School of Medicine, New York, NY 10029. Dr. Jacobs' present address is Department of Medicine, Boston University School of Medicine, Boston, MA 02215.

Received for publication 11 February 1982 and in revised form 30 June 1982. the high carbohydrate-SRIF periods. These values were not significantly different. Postprandial glucose levels were slightly increased during high carbohydrate-SRIF, but overnight glucose concentrations were not affected. Plasma FFA levels were not different during the two high carbohydrate periods. Plasma glucagon levels did not appear to affect the results either. This study indicates that postprandial hyperinsulinemia during a high carbohydrate diet is not necessary for induction of hypertriglyceridemia.

\section{INTRODUCTION}

During the past two decades, several groups of investigators have presented evidence indicating a close relationship between plasma insulin levels and fasting plasma triglyceride levels. Significant positive correlations have been demonstrated between both fasting and postprandial plasma insulin concentrations and the fasting concentration of plasma triglyceride in subjects with normal plasma triglyceride levels (1) and in subjects with hypertriglyceridemia (2-4). Carbohydrate induction of hypertriglyceridemia has been used as a model of the insulin-triglyceride relationship (5, 6). During consumption of a fat-free, $75-85 \%$ carbohydrate diet, plasma triglyceride levels increase and this rise has been found to result from an increase in very low density lipoprotein (VLDL)-triglyceride secretion into plasma (6-8). Postprandial insulin responses during consumption of this diet have been found to correlate with VLDL-triglyceride production rates (6). However, only a single study has been published in which there was an attempt to directly study the role of plasma insulin levels in the regulation of VLDLtriglyceride production (9). 
Somatostatin (SRIF) ${ }^{1}$ is a tetradecapeptide which is present in many organs, including the hypothalamus and the pancreas. SRIF is a potent inhibitor of the secretion of many hormones, including insulin (10). We chose, therefore, to utilize SRIF as a tool for directly probing the role of the postprandial insulin response in carbohydrate-induced hypertriglyceridemia.

\section{METHODS}

Eight subjects with a range of fasting plasma triglyceride levels were studied. The clinical characteristics of the group are presented in Table I. All subjects had normal fasting plasma glucose concentrations and none had any diseases known to result in secondary hyperlipidemia. No subject was taking any medication known to affect lipid or carbohydrate metabolism. All subjects gave informed consent before entering the study.

Protocols. Each subject was admitted to the Clinical Research Center at the University Hospital of the University of California at San Diego School of Medicine, La Jolla, CA. The subjects remained in the Center throughout the entire study period. Physical activity was limited to walking about the Center. Routine blood chemistries, complete blood count, coagulation profile, and urinalysis were obtained. For the first $7 \mathbf{d}$ (base-line period), the subjects were fed a weightmaintaining liquid formula diet $(30-35 \mathrm{kcal} / \mathrm{kg}$ per d) consisting of $40 \%$ carbohydrate, $40 \%$ fat, $20 \%$ protein with a ratio of polyunsaturated fat equal to 2.0 , and $300 \mathrm{mg}$ cholesterol daily. The diet was given in four equally divided meals at $900,1200,1500$, and $1800 \mathrm{~h}$. After this base-line period, subjects 1-5 were switched to a high carbohydrate liquid formula diet consisting of $80 \%$ carbohydrate and $20 \%$ protein, which was consumed at the same times used during the base-line period. This fat-free formula was consumed for the next $5 \mathrm{~d}$ (high carbohydrate period). During the succeeding $48 \mathrm{~h}$, the turnover of VLDL-triglyceride was measured (see below). For this period, subjects were given the same fat-free formula, but received only $60 \%$ of the total daily calories used to maintain their weight. In addition, they now ingested the formula every $3 \mathrm{~h}$. This protocol has been shown to maintain steady-state plasma triglyceride concentrations throughout the $48 \mathrm{~h}$ of the VLDL-triglyceride turnover study period while eliminating the periodic influx of chylomicrons that would result from consumption of dietary fat $(8,11)$. After completion of the 48 -h turnover protocol, the subjects returned to the base-line period diet for another $7 \mathrm{~d}$. This was followed by a second 5-d period of the isocaloric $80 \%$ carbohydrate, $20 \%$ protein formula diet. However, during this period, an intravenous infusion of SRIF $(1.3 \mu \mathrm{g} / \mathrm{min}$ in $250 \mathrm{ml}$ of $0.15 \mathrm{M} \mathrm{NaCl})$ was given daily between 800 and $2100 \mathrm{~h}$ (via a portable pump; IVAC Corp., San Diego, CA) to inhibit postprandial hyperinsulinemia (high carbohydrate-SRIF period). SRIF was kindly supplied by Dr. M. Brown and Dr. R. Guillerman of the Salk Institute (San Diego, CA). At the end of this 5-d period, the subjects underwent a second 48-h VLDL-triglyceride turnover protocol. No SRIF was given during the 48-h period and the two turnover studies were therefore carried out under identical circumstances. This approach was taken so that we might directly compare the effects of each 5 -d period preceding the turnover protocols. Subjects 6-8 underwent the

\footnotetext{
${ }^{1}$ Abbreviations used in this paper: FCR, fractional catabolic rate; SRIF, somatostatin.
}

TABLE I

Clinical Characteristics

\begin{tabular}{cccccc}
\hline Subject & Age & Sex & IBW & TG 1 & Cholesterolt \\
\hline & & & $\%$ & & \\
1 & 56 & M & 105 & 358 & 222 \\
2 & 61 & M & 118 & 149 & 208 \\
3 & 38 & M & 132 & 263 & 208 \\
4 & 59 & F & 115 & 190 & 227 \\
5 & 50 & M & 105 & 244 & 272 \\
6 & 60 & M & 112 & 100 & 261 \\
7 & 55 & F & 134 & 160 & 268 \\
8 & 55 & M & 102 & 271 & 241 \\
\hline
\end{tabular}

- Percent ideal body weight (IBW) based on the Metropolitan Life Insurance Tables.

\ Fasting plasma triglyceride (TG) and cholesterol levels (milligrams per deciliter) at the start of the first base-line diet period.

identical study except that the high carbohydrate-SRIF period preceded the high carbohydrate period. Body weights of all the subjects remained within $2 \%$ of their admission weights throughout the entire study period.

Blood sampling. Fasting plasma triglyceride levels were measured daily throughout the four study periods. On day 6 and 7 of the first base-line period and on day 4 and 5 of each of the high carbohydrate periods (with/without SRIF) blood was taken for measurement of plasma glucose, insulin, and glucagon at 1,2 , and $3 \mathrm{~h}$ after both the 900- and 1800 $h$ feedings. In addition, three subjects had blood samples drawn for measurement of these three substances every 3 $\mathrm{h}$ for the $48 \mathrm{~h}$ encompassing each of these 2-d sampling periods. Plasma FFA levels were also measured every $3 \mathrm{~h}$ over the 2-d sampling period during the high carbohydrate and the high carbohydrate-SRIF periods in these three subjects (FFA levels were not measured during the base-line period).

All blood samples, except those for glucagon determination, were drawn into EDTA tubes $(1.0 \mathrm{mg} / \mathrm{ml})$ and centrifuged at $1,500 \mathrm{~g}$ for $30 \mathrm{~min}$. The plasma was separated and placed at $4^{\circ} \mathrm{C}$ for lipid and lipoprotein measurements or at $-20^{\circ} \mathrm{C}$ until assay for glucose, insulin, and FFA levels. Blood for measurement of plasma glucagon was put into tubes containing Trasylol (Delbay Pharmaceuticals Inc., Div. Schering Corp., Bloomfield, NJ). Plasma was quickly separated and stored at $-70^{\circ} \mathrm{C}$ until assay. Plasma immunoreactive insulin was determined by radioimmunoassay (12) with ${ }^{125}$ I insulin purchased from New England Nuclear (Boston, MA) and an antiporcine insulin antibody obtained from Miles Laboratory Inc., Elkhart, IN. Insulin used as standard was from Novo Research Institute, Copenhagen, Denmark. Plasma glucose was measured by using glucose oxidase and a Beckman Analyzer (Beckman Instruments, Fullerton, CA). Plasma-immunoreactive glucagon was determined by using the method of Unger (13). ${ }^{125}$ I-Glucagon was purchased from New England Nuclear, the antiglucagon antibody $(30,000 \mathrm{~mol} \mathrm{wt})$ was obtained from the laboratory of Dr. R. Unger, and standard glucagon was obtained from Lilly (Eli International Corporation, Indianapolis, IN). Plasma FFA levels were measured by the method of Noma et al. (14). Plasma triglyceride and cholesterol were measured by Lipid Research Clinic methods using the Technicon autoanalyzer (Technicon Instruments Corp., Tarrytown, NY) (15). Measurement 
of the plasma turnover of VLDL-triglycerides were carried out using the protocol of Grundy et al. (11). 18 blood samples were taken during the 48-h period following injection of 300 $\mu \mathrm{Ci}$ of $\left[2{ }^{3} \mathrm{H}\right] \mathrm{glycerol}$. VLDL $(d=1.006)$ was isolated from each of the 18 plasma samples by ultracentrifugation at $39,000 \mathrm{rpm} \times 20 \mathrm{~h}$ at $10^{\circ} \mathrm{C}$ in a 40.3 rotor using a Beckman L5-65 ultracentrifuge (Beckman Instruments). Aliquots of each sample were used for measurement of triglyceride and for determination of $\left[{ }^{3} \mathrm{H}\right]$ triglyceride radioactivity. The resulting specific radioactivity curve for VLDL- $\left.-{ }^{3} \mathrm{H}\right]$ triglyceride was used to determine the fractional clearance rate (FCR) of the VLDL from plasma $(11,16)$. The FCR multiplied by the VLDL-triglyceride pool during the steady state turnover period yields the production rate of the lipoprotein lipid.

Statistical analysis was carried out using the $t$ test for paired data. Each subject's data obtained during the first base-line period were compared with those obtained during the high carbohydrate periods (with and without SRIF). In addition, the data from the two high carbohydrate periods were compared with each other. Because of wide subject variability in the absolute levels of the measured substances, the postprandial data were plotted as percentage of baseline value. The three hourly samples obtained over a $48-\mathrm{h}$ period were analyzed by analysis of variance for effect of time and diet (17). Correlation coefficients were calculated using linear regression analysis.

\section{RESULTS}

Fig. 1 depicts the mean summed insulin response of the group 1,2 , and $3 \mathrm{~h}$ after the 900 - and 1800 -h feedings during the high carbohydrate and high carbohydrate-SRIF periods (mean of data from days 4 and 5 of each diet period). The data is depicted as the percent change from the base-line period response.

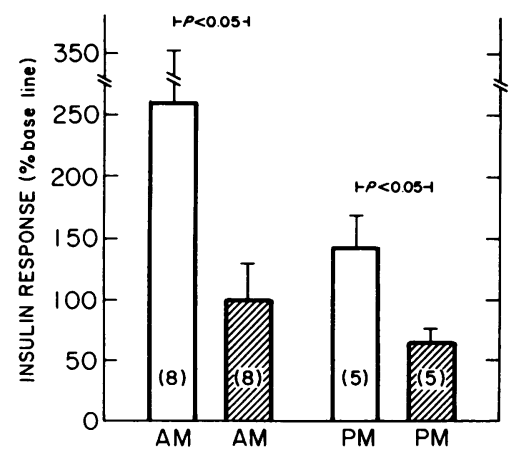

FIGURE 1 Effect of SRIF infusion on postprandial insulin responses to a high carbohydrate diet. The summed insulin responses for the group (number of subjects studied are in parentheses) 1,2 , and $3 \mathrm{~h}$ after the $900 \mathrm{~h}$ (AM) and 1800 $h(P M)$ formula feedings during the high carbohydrate (open bars) and high carbohydrate-SRIF (hatched bars) periods are depicted. The responses are presented as percent of response ( $\pm \mathrm{SE}$ ) during the base-line period. Each subject's response in each period was the mean of their responses on the last $2 \mathrm{~d}$ of that period. The $P$ values indicate statistically significant differences between the responses in the two high carbohydrate periods. Additionally, the responses during the high carbohydrate period (open bars) were significantly greater than during base line $(P<0.05)$.
Compared with the base-line period, there was a $161 \%$ increase in the insulin response to the 900 - $h$ feeding, and a $35 \%$ increase in the response to the 1800 - $h$ feeding during the high carbohydrate diet period. The addition of the SRIF infusion between 800 and 2100 $\mathrm{h}$ completely abolished the hyperinsulinemic response to the high carbohydrate formula feedings.

The effect of the SRIF infusion on the plasma glucose response during the two feeding periods is depicted in Fig. 2. The glucose responses to the high carbohydrate formula alone at 900 and $1800 \mathrm{~h}$ were not different from the responses to the base-line diet. The glucose response during high carbohydrate-SRIF was increased by $\sim 50 \%$ after the morning feeding, but was not significantly higher than base line after the evening feeding. Thus, suppression of the hyperinsulinic response to high carbohydrate by SRIF was associated with modest increases in plasma glucose levels in the morning but not in the evening. Plasma glucagon levels in both postmeal periods were, as expected, suppressed by the high carbohydrate diet compared with base line (Fig. 3). Although both the AM and PM responses during high carbohydrate-SRIF were significantly decreased vs. the base-line period responses, only the $\mathrm{AM}$ response during the former was significantly lower than that response during high carbohydrate alone. The individual AM and PM postprandial responses of plasma glucose, insulin, and glucagon during each study period are presented in Table II.

To demonstrate that the effect of SRIF was uniform throughout the day and to assure ourselves that rebound elevations of hormones and substrates did not occur when the SRIF infusions were stopped, three subjects (6-8) had, in addition, blood samples drawn every $3 \mathrm{~h}$ during the last $48 \mathrm{~h}$ of the base-line period

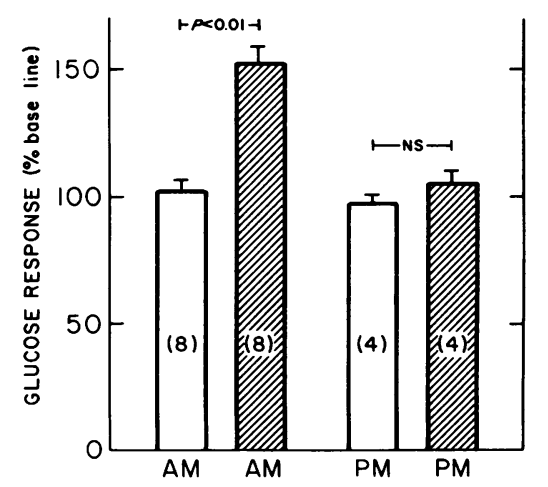

FIGURE 2 Effect of SRIF infusion on postprandial glucose response to a high carbohydrate diet. The data were obtained as described in Fig. 1. The AM response during high carbohydrate-SRIF (hatched bar) was also greater than during base line $(P<0.05)$. 


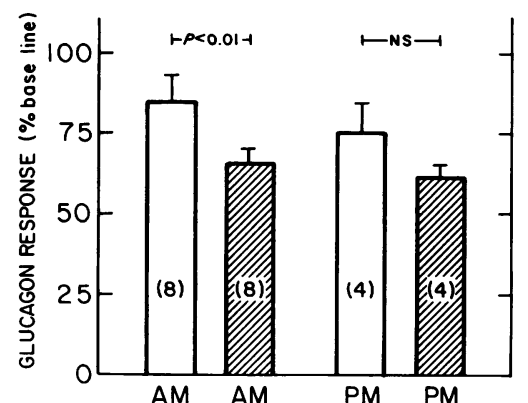

FIGURE 3 Effect of SRIF infusion on postprandial glucagon response to a high carbohydrate diet. The data were obtained as described in Fig. 1. Both the AM and PM responses during high carbohydrate-SRIF (hatched bars) were less than base line $(P<0.05)$.

and each diet period. The profiles for plasma insulin, glucose, and glucagon were analyzed for effect of time and diet (with/without SRIF). Insulin and glucose levels were strongly affected by time, with diurnal variations evident. In addition, daytime levels of all three substances were affected by the administration of SRIF in a manner similar to that seen during the immediate postprandial periods (Figs. 1-3). Thus, plasma insulin and glucagon concentrations were reduced and plasma glucose levels increased during infusion of SRIF compared with base line. However, these data also indicated that overnight concentrations of all three of these

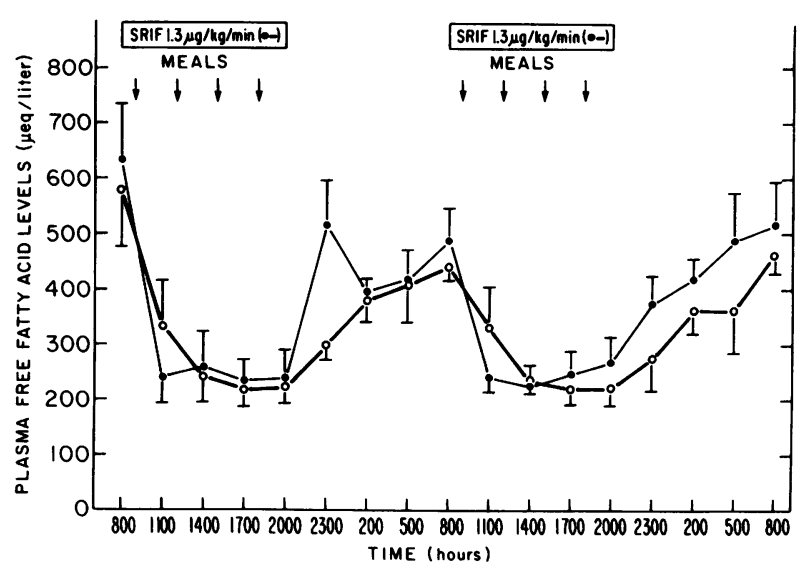

Figure 4 Effect of SRIF infusion on plasma FFA levels. Three subjects had FFA measured every $3 \mathrm{~h}$ for $48 \mathrm{~h}$ during the high carbohydrate (closed circles) and high carbohydrate-SRIF (open circles) periods. Formula feedings were consumed at the times indicated by the arrows. Analysis of variance revealed a significant effect of time $(P<0.001)$ upon FFA levels, but no effect of high carbohydrate-SRIF vs. high carbohydrate alone. Data are presented as mean $\pm \mathrm{SE}$.

substances were the same during the base line and the two study periods. The FFA data from the two high carbohydrate periods are depicted in Fig. 4. Although there was an obvious diurnal rhythm of plasma FFA levels, SRIF infusion had no effect on the FFA concentrations in these subjects.

TABLE II

Individual Postprandial Insulin, Glucose, and Glucagon Responses

\begin{tabular}{|c|c|c|c|c|c|c|c|c|c|}
\hline \multirow[b]{2}{*}{ Subject } & \multicolumn{3}{|c|}{ Glucose } & \multicolumn{3}{|c|}{ Insulin } & \multicolumn{3}{|c|}{ Glucagon } \\
\hline & At & Bt & $\mathrm{Cl}$ & At & B! & $\mathrm{Ct}$ & At & $\mathrm{Bt}$ & $\mathrm{Ct}$ \\
\hline \multicolumn{10}{|c|}{ AM Postprandial ${ }^{\circ}$} \\
\hline 1 & 432 & 499 & 545 & 243 & 547 & 123 & 449 & 299 & 241 \\
\hline 2 & 361 & 300 & 507 & 444 & 677 & 166 & 375 & 341 & 248 \\
\hline 3 & 316 & 415 & 423 & 105 & 965 & 350 & 496 & 491 & 377 \\
\hline 4 & 300 & 272 & 587 & 106 & 140 & 63 & 592 & 339 & 373 \\
\hline 5 & 504 & 462 & 538 & 324 & 380 & 128 & 586 & 380 & 325 \\
\hline 6 & 327 & 432 & 539 & 177 & 305 & 138 & 641 & 575 & 409 \\
\hline 7 & 314 & 312 & 566 & 332 & 406 & 264 & 890 & 1176 & 811 \\
\hline 8 & 314 & 291 & 518 & 136 & 204 & 189 & 687 & 742 & 475 \\
\hline \multicolumn{10}{|c|}{ PM Postprandial ${ }^{\circ}$} \\
\hline 1 & 366 & 396 & 513 & 132 & 154 & 102 & 439 & 416 & 297 \\
\hline 2 & 318 & 295 & 314 & 258 & 402 & 132 & 411 & 366 & 256 \\
\hline 3 & 386 & 381 & 361 & 211 & 419 & 139 & 565 & - & 379 \\
\hline 4 & 334 & 277 & 374 & 114 & 70 & 45 & 542 & 320 & 347 \\
\hline 5 & 294 & 392 & - & 136 & 253 & 128 & 713 & 429 & 339 \\
\hline
\end{tabular}

- AM, sum of hourly plasma levels at 1000,1100 , and $1200 \mathrm{~h}$; PM, sum of hourly plasma levels at 1900,2000 , and $2100 \mathrm{~h}$.

I A, base line diet; B, high carbohydrate alone; C, high carbohydrate plus SRIF. All values are means of responses on days 4 and 5 of each diet period. 
The individual responses of plasma triglyceride levels and of VLDL-triglyceride synthesis to the high carbohydrate formula with or without concomitant SRIF infusion are presented in Table III. It is clear that the plasma triglyceride concentrations in each subject were comparable at the end of each base-line period. Thus, their responses to the two high carbohydrate periods can be directly compared. There was a variable effect of SRIF infusion upon the increase of plasma triglycerides during the high carbohydrateSRIF period. Four subjects had a greater increase during the high carbohydrate-SRIF period, whereas four subjects demonstrated greater induction of hypertriglyceridemia during the high carbohydrate period. There was no difference between the mean plasma triglyceride concentration for the group during the two periods (with/without SRIF). The similar degrees of carbohydrate-induced hypertriglyceridemia were associated with similar mean VLDL production rates for the group during the two high carbohydrate diet periods. Four of the seven subjects studied actually had higher rates of VLDL-triglyceride secretion during the high carbohydrate-SRIF period. Two subjects had the same secretion rates and one had a decreased rate of VLDL-triglyceride production during high carbohydrate-SRIF. Finally, postheparin plasma lipoprotein lipase and hepatic triglyceride lipase activities were unaffected by daily SRIF infusions (data not shown).

As noted by other workers (1-6), there were correlations between the postprandial plasma insulin response (AM) and the fasting plasma VLDL-triglyceride concentration during both the base-line period $(r=0.65)$ and the high carbohydrate period $(r=0.64)$, although these values did not reach statistical significance at the level of $P<0.5$. This relationship disappeared when postprandial insulin levels were suppressed during the high carbohydrate-SRIF period ( $r$ $=0.05$ ), although an equivalent degree of hypertriglyceridema was induced. The degree of suppression

TABLE III

Effect of SRIF on Carbohydrate-induced Hypertriglyceridemia

\begin{tabular}{|c|c|c|c|c|c|}
\hline \multirow[b]{2}{*}{ Subject } & \multirow[b]{2}{*}{ Base line ${ }^{\circ}$} & \multicolumn{2}{|c|}{ Triglyceride ${ }^{\circ}$} & \multicolumn{2}{|c|}{ VLDI-TG-SR $\rfloor$} \\
\hline & & $\mathrm{Hi}-\mathrm{CHO} \S$ & Hi-CHO-SRIF $\$$ & $\mathrm{Hi} \cdot \mathrm{CHO} \$$ & Hi-CHO-SRIF $\$$ \\
\hline 1 & $\begin{array}{l}358 \\
352\end{array}$ & 439 & 639 & 19.1 & 28.9 \\
\hline 2 & $\begin{array}{l}149 \\
149\end{array}$ & 421 & 444 & 27.0 & 45.7 \\
\hline 3 & $\begin{array}{l}263 \\
260\end{array}$ & 834 & 658 & 21.0 & 30.6 \\
\hline 4 & $\begin{array}{l}190 \\
190\end{array}$ & 379 & 512 & 32.9 & 34.3 \\
\hline 5 & $\begin{array}{l}244 \\
232\end{array}$ & 458 & 512 & 25.7 & 102.0 \\
\hline $6^{\|}$ & $\begin{array}{l}100 \\
110\end{array}$ & 308 & 287 & 30.1 & 21.0 \\
\hline $7^{\prime \prime}$ & $\begin{array}{l}160 \\
147\end{array}$ & 383 & 241 & 23.1 & 24.2 \\
\hline $8^{\prime \prime}$ & $\begin{array}{l}233 \\
297\end{array}$ & 585 & 563 & - & - \\
\hline Mean \pm SD & & $476 \pm 165$ & $482 \pm 152$ & $25.6 \pm 4.9$ & $40.9 \pm 28.1$ \\
\hline
\end{tabular}

- Mean fasting plasma triglyceride concentration (milligrams per deciliter) during the last $3 \mathrm{~d}$ of each diet period. Values for both base-line periods are shown for each subject. I VLDL-TG-SR, very low density lipoprotein triglyceride secretion rate (milligrams per killigrams per hour).

$\S \mathrm{Hi}-\mathrm{CHO}$, high carbohydrate diet; Hi-CHO-SRIF, high carbohydrate diet and SRIF infusion between 800 and $2100 \mathrm{~h}$.

"Subjects 6-8 received $\mathrm{Hi}-\mathrm{CHO}-\mathrm{SRIF}$ before $\mathrm{Hi}-\mathrm{CHO}$. 
TABLE IV

Effect of SRIF with/without Glucagon Replacement on

Carbohydrate-induced Hypertriglyceridemia

\begin{tabular}{cccccc}
\hline Subject & Base line & Hi-CHOt & Hi-CHO-SRIFt & Base line & \begin{tabular}{c} 
Hi $+\begin{array}{c}\text { CHO-SRIF } \\
+ \text { glucagon }\end{array}$ \\
\hline 1
\end{tabular} \\
\hline 3 & 355 & 439 & 639 & 188 & 709 \\
3 & 261 & 834 & 658 & 232 & 732 \\
\hline
\end{tabular}

- Mean of two base-line periods during the Hi-CHO and Hi-CHO-SRIF studies (milligrams per deciliter) (see Table III).

I Mean of the plasma triglyceride concentrations during the final $3 \mathrm{~d}$ of each study period.

$\S$ Plasma triglyceride during the third base-line period preceding the glucagon replacement study. Mean of the last $2 \mathrm{~d}$ of that period.

of postprandial insulin secretion by SRIF in each individual, calculated as either the fraction of the baseline postprandial insulin response or the fraction of the high carbohydrate diet postprandial insulin response present during high carbohydrate-SRIF, was not correlated with the rise in VLDL-triglyceride concentration or the secretion rate of VLDL-triglyceride during the latter period.

Because plasma glucagon levels were suppressed more during the high carbohydrate-SRIF period than during the high carbohydrate period, two subjects underwent a third study at a later date. After another 7-d base-line diet period, each received the same high carbohydrate formula and SRIF infusion as before, but, in addition, each received an intravenous infusion of glucagon $(1.0 \mathrm{ng} / \mathrm{kg}$ per $\mathrm{min}$ ) between 800 and $2100 \mathrm{~h}$ daily for the 5 -d study period. Table IV indicates that the degree of hypertriglyceridemia induced by the high carbohydrate-SRIF protocol was at

TABLE V

Postprandial Glucagon Responses during the High Carbohydrate Diet Alone and during High Carbohydrate Diets with SRIF and with SRIF Plus Glucagon

\begin{tabular}{|c|c|c|c|c|c|c|}
\hline \multirow[b]{2}{*}{ Subject } & \multicolumn{2}{|c|}{$\mathrm{Hi-CHO}$} & \multicolumn{2}{|c|}{ Hi-CHO-SRIF ${ }^{\bullet}$} & \multicolumn{2}{|c|}{$\begin{array}{l}\text { Hi-CHO-SRIF- } \\
\text { Glucagon }\end{array}$} \\
\hline & AMI & PMI & AMI & PMI & AMI & PMI \\
\hline 1 & 299 & 416 & 241 & 297 & 551 & 762 \\
\hline 3 & 491 & - & 377 & 379 & 890 & 776 \\
\hline
\end{tabular}

- Hi-CHO, high carbohydrate alone; Hi-CHO-SRIF, high carbohydrate plus somatostatin $(1.3 \mu \mathrm{g} / \mathrm{min})$; Hi-CHO-SRIF-Glucagon, high carbohydrate plus somatostatin $(1.3 \mu \mathrm{g} / \mathrm{min})$ plus glucagon (1.0 $\mathrm{ng} / \mathrm{kg}$ per $\mathrm{min}$ ).

$\$ \mathrm{AM}$, sum of hourly plasma glucagon levels at 1000,1100 , and $1200 \mathrm{~h}$; PM, sum of hourly plasma glucagon levels at 1900,2000 , and $2100 \mathrm{~h}$. least as great during replacement of glucagon as during the period when glucagon levels were suppressed. The plasma glucagon levels during replacement were significantly greater than those present during either of the high carbohydrate periods (with and without SRIF) (Table V).

\section{DISCUSSION}

Our goal in this investigation was to test directly the hypothesis that postprandial hyperinsulinemia was causally related to the hypertriglyceridemia associated with ingestion of high carbohydrate diets. To achieve this goal, we used SRIF, a potent inhibitor of the secretion of several peptide hormones, to inhibit the increased postprandial insulin response to an isocaloric, fat-free, high carbohydrate diet in a group of subjects with a wide range of fasting plasma triglyceride levels. Under the conditions of our protocol, SRIF abolished the excessive postprandial insulin response to a high carbohydrate diet without causing absolute insulin deficiency. In addition, overnight plasma insulin levels were unaffected by the daytime infusion of SRIF. Although postprandial hyperinsulinemia was prevented during high carbohydrate-SRIF, induction of hypertriglyceridemia was essentially identical in our eight subjects during that period, compared with the high carbohydrate alone period when postprandial insulin levels were significantly increased vs. base line.

These data indicate that although elevated postprandial plasma insulin levels may be associated with the elevated plasma triglyceride concentrations that occur during consumption of a high carbohydrate diet (5-8), a causal relationship need not exist. Previous attempts to test the hypothesis relating plasma insulin and triglyceride levels have been limited. Olefsky et al. demonstrated that weight loss resulted in a decrease in postprandial plasma insulin and fasting triglyceride levels as well as a decrease in VLDL-triglyceride pro- 
duction (18). However, although that study demonstrated constancy of the relationships between these three variables before and after weight loss, the many other metabolic changes accompanying weight loss, e.g., an improvement in insulin sensitivity $(18,19)$, restrict interpretation of the data. A more direct approach was taken by Eaton and Nye (9), who administered diazoxide, an agent that suppresses insulin secretion, to four subjects with a wide range of fasting plasma triglyceride concentrations. All four subjects had decreases in fasting plasma triglyceride concentrations during the 5-7 d of diazoxide administration. Although "overt glucose intolerance" was noted in all four patients during the study period, no quantitative data were given. In addition, no data regarding plasma glucagon or ketone levels or degree of glucosuria were included. However, this report did suggest that some critical level of insulin action might be necessary to maintain triglyceride secretion.

Evidence in support of a critical role for insulin in the synthesis and secretion of triglycerides by the liver can also be found in studies using animal models of diabetes mellitus. Thus, several groups have demonstrated markedly reduced secretion of VLDL-triglyceride in chemically or surgically induced diabetes mellitus in rats $(20,21)$ and dogs $(22)$. Insulin secretion was markedly reduced in these animal models. In contrast, in most studies of human diabetes mellitus, increased plasma triglyceride concentrations have been associated with increased production of VLDL-triglyceride $(23,24){ }^{2.3}$ Overall, these studies of triglyceride metabolism in diabetes mellitus strongly suggest that a minimal level of insulin is necessary to maintain synthetic and secretory mechanisms for VLDL-triglyceride production, but that in the presence of this critical level of insulin, increased availability of substrates such as glucose and FFA can result in increased synthesis of triglyceride. This view is supported by in vitro work using perfused or perifused liver cells in which addition of superphysiologic quantities of insulin to insulin-free media increased release of triglyceride into the media $(25,26)$. In the present study, although postprandial insulin secretion was inhibited by SRIF, insulin concentrations during infusion of SRIF were equal to or only slightly less than those present during the base-line period. Thus, absolute insulin deficiency was never present in our subjects. This fact differentiates our study from that of Eaton and

\footnotetext{
${ }^{2}$ Abrams, J. J., H. Ginsberg, and S. M. Grundy. Metabolism of cholesterol and plasma triglycerides in nonketotic diabetes mellitus. Diabetes. In press.

${ }^{3}$ Ginsberg, H., and S. M. Grundy. Very low density lipoprotein metabolism in non-ketotic diabetes mellitus: Effect of caloric restriction. Diabetologia. In press.
}

Nye (9) and from the animal studies mentioned (20-22).

Several alternative explanations of our results must be addressed. First, inhibition of the postprandial plasma insulin response to the high carbohydrate diet by the infusion of SRIF was associated with increased plasma glucose levels. However, this was only significant during the hours immediately following the feeding at $900 \mathrm{~h}$. In addition, overnight glucose levels, measured in three subjects, were the same in both high carbohydrate periods and did not differ from those present during the base-line period. Thus, although we concede that modest increases in plasma glucose levels, as an indication of diminished postprandial insulin secretion, might have provided increased substrate for triglyceride production, we do not believe this can account for the degree of hypertriglyceridemia that occurred during the high carbohydrate-SRIF period.

Because we expected that postprandial glucagon levels would be significantly suppressed during the high carbohydrate diet (without SRIF), we did not infuse glucagon along with SRIF in our original protocol. However, in most studies, plasma glucagon levels were further suppressed during high carbohydrate-SRIF. Because glucagon is an important regulator of hepatic FFA metabolism, with decreased glucagon levels associated with increased rates of FFA incorporation into triglycerides $(27,28)$, we further investigated the role of glucagon suppression during infusion of SRIF. First, in the three subjects sampled for $48 \mathrm{~h}$, overnight glucagon levels did not differ in any of the three sampling periods (including base line). Thus, there was no evidence to suggest that hypoglucagonemia might have resulted in increased incorporation of FFA into hepatic triglyceride during the nighttime hours of the high carbohydrate-SRIF period. More direct evidence against a significant role for hypoglucagonemia in these studies resulted from our glucagon-reinfusion studies. During high carbohydrate-SRIF plus glucagon, when plasma glucagon levels were maintained at levels greater than those present during either high carbohydrate period, hypertriglyceridemia developed to the same degree as that present during the high carbohydrate period in the two subjects studied. Although a peripheral infusion of glucagon may not perfectly mimic the secretion of that hormone into the portal vein, the levels we attained in the periphery were great enough to mimic the portal vein concentrations present during the high carbohydrate period (29). We believe, therefore, that the glucagon reinfusion studies eliminate the possibility that the increased inhibition of glucagon secretion observed during high carbohydrate-SRIF played a significant role in the induction of hypertriglyceridemia observed during that period. 
FFA are the major substrate regulating hepatic triglyceride synthesis $(30,31)$. Thus, in liver perfusion studies, for any levels of plasma insulin present, increasing the FFA levels results in increasing triglyceride synthesis (31). Overnight levels of FFA are thought to be important regulators of the response of fasting plasma triglycerides to a high carbohydrate diet (2). In fact, Shlierf et al. demonstrated inhibition of carbohydrate-induced hypertriglyceridemia by overnight intravenous infusions of glucose, which suppressed nocturnal flux of FFA (32). In the three subjects studied, overnight plasma FFA levels did not differ during the two study periods. Thus, although flux of plasma FFA was not measured directly, it is probable that FFA transport to the liver was similar during both high carbohydrate periods.

Finally, the use of SRIF as a probe of the insulintriglyceride relationship may itself have introduced an artifact. SRIF has been shown to diminish absorption of glucose from the small intestine (33). However, this would not have had major significance in our study, where the response of the liver to a high carbohydrate diet is slow, requiring several days to reach equilibrium $(5,6)$. Direct effects of SRIF on hepatocyte triglyceride secretion or on FFA release from adipocytes have not been reported. In this regard, we have measured plasma triglycerides in four subjects over two 72-h periods during which they consumed an isocaloric diet of $40 \%$ carbohydrate, $40 \%$ fat, and $20 \%$ protein with and without a simultaneous, continuous infusion of SRIF. No effect of SRIF on plasma triglycerides was noted (unpublished observation).

In summary, the data presented here demonstrate that postprandial increases in insulin are not necessary for the induction of hypertriglyceridemia by high carbohydrate diets. Thus, although highly significant correlations can be found between postprandial insulin responses and rates of VLDL-triglyceride production, no direct causal relationship need exist. A coexistent abnormality that affects the regulation of these two variables could explain their close association. Such a common factor might be cellular resistance to the effects of insulin on glucose metabolism and lipolysis $(2,34)$. This abnormality would result in decreased glucose oxidation and increased FFA flux in the face of normal or elevated plasma insulin levels and would provide an excellent milieu for the synthesis of VLDLtriglyceride. Finally, although the present study did not directly address the issue, the significant relationships demonstrated between elevated levels of fasting plasma insulin and hypertriglyceridemia may similarly only reflect the common association of these two states with a metabolic defect that is causally linked to both.

\section{REFERENCES}

1. Tobey, T. A., M. Greenfield, F. Kraemer, and G. M. Reaven. 1981. Relationship between insulin resistance, insulin secretion, very low density lipoprotein kinetics and plasma triglyceride levels in normotriglyceridemic man. Metab. Clin. Exp. 30: 165-171.

2. Olefsky, J. M., J. W. Farquhar, and G. M. Reaven. 1974. Reappraisal of the role of insulin in hypertriglyceridemia. Am. J. Med. 57: 551-560.

3. Bierman, E. L. 1972. Insulin and hypertriglyceridemia. Isr. J. Med. Sci. 8: 304-308.

4. Tzagournis, M., R. Chiles, J. M. Ryan, and T. G. Skillman. 1968. Interrelationships of hyperinsulinemia and hypertriglyceridemia in young patients with coronary heart disease. Circulation. 38: 1156-1163.

5. Farquhar, J. W., A. Frank, R. C. Gross, and G. M. Reaven. 1966. Glucose, insulin, and triglyceride response to high and low carbohydrate diets in man. J. Clin. Invest. 45: 1648-1656.

6. Reaven, G. M., R. L. Lerner, M. P. Stern, and J. W. Farquhar. 1967. Role of insulin in endogenous hypertriglyceridemia. J. Clin. Invest. 46: 1756-1767.

7. Quarfordt, S. H., A. Frank, D. M. Shames, M. Berman, and D. Steinberg. 1970. Very low density lipoprotein triglyceride transport in type IV hyperlipoproteinemia and the effects of carbohydrate-rich diets. J. Clin. Invest. 49: 2281-2297.

8. Melish, J., N. A. Le, H. Ginsberg, D. Steinberg, and W. V. Brown. 1980. Dissociation of apoprotein B and triglyceride production in very low density lipoproteins. Am. J. Physiol. 239: E354-E362.

9. Eaton, P., and W. H. R. Nye. 1975. The relationship between insulin secretion and triglyceride concentration in endogenous lipemia. J. Lab. Clin. Med. 81: 682-695.

10. Vale, W., C. Rivier, and M. Brown. 1977. Regulatory peptides of the hypothalamus. Annu. Rev. Physiol. 29: 473-527.

11. Grundy, S. M., H. Y. I. Mok, L. Zech, D. Steinberg, and M. Berman. 1979. Transport of very low density lipoprotein triglycerides in varying degrees of obesity and hypertriglyceridemia. J. Clin. Invest. 63: 1274-1283.

12. Desbuquois, B., and G. D. Aurback. 1971. Use of polyethylene glycol to separate free from antibody bound peptide hormones in radioimmunoassays. J. Clin. Endocrinol. Metab. 33: 732-738.

13. Faloona, G. R., and R. H. Unger. 1974. Glucagon. In Methods of Hormone Radioimmunoassays. B. M. Jaffe and H. R. Berman, editors. Academic Press, Inc., New York. 317-330.

14. Noma, A., H. Okabe, and M. Keta. 1973. A new colorimetric microdetermination of free fatty acids in serum. Clin. Chim. Acta. 43: 317-320.

15. Lipid Research Clinic Program. 1974. Lipid and lipoprotein analysis. In Manual of Laboratory Operations. Department of Health, Education, and Welfare Publications No. NIH 75-628. Washington, DC.

16. Le, N. A., S. M. Grundy, and M. Berman. 1982. A simplified model of very low density lipoprotein triglyceride metabolism. In Lipoprotein Kinetics and Modeling. M. Berman, S. M. Grundy, and B. Howard, editors. Academic Press, Inc., New York. In press.

17. SAS User's Guide. 1979. SAS Institute, Inc. Raleigh, NC.

18. Olefsky, J., G. M. Reaven, and J. W. Farquhar. 1974. Effects of weight reduction on obesity. Studies of lipid 
and carbohydrate metabolism in normal and hyperlipoproteinemic subjects. J. Clin. Invest. 53: 64-76.

19. Drenick, E. J., A. S. Brickman, and E. M. Gold. 1972. Dissociation of the obesity-hyperinsulinism relationship following dietary restriction and hyperalimentation. Am. J. Clin. Nutr. 25: 746-751.

20. Reaven, E. P., and G. M. Reaven. 1974. Mechanisms for development of diabetic hypertriglyceridemia in streptozotocin-treated rats. J. Clin. Invest. 54: 1167-1178.

21. Van Tol, A. 1977. Hypertriglyceridemia in the diabetic rat. Defective removal of serum very low density lipoproteins. Atherosclerosis. 26: 117-128.

22. Basso, L. V., and R. J. Havel. 1974. Hepatic metabolism of free fatty acids in normal and diabetic dogs. J. Clin. Invest. 49: 537-547.

23. Nikkila, E. A., J. K. Huttinen, and C. Ehnholm. 1977. Postheparin plasma lipoprotein lipase and hepatic lipase in diabetes mellitus: relationship to triglyceride metabolism. Diabetes. 26: 11-21.

24. Greenfield, M., O. Kolterman, J. Olefsky, and G. M. Reaven. 1980. Mechanism of hypertriglyceridemia in diabetic patients with fasting hyperglycemia. Diabetologia. 18: 441-446.

25. Topping, D. L., and P. A. Mayes. 1972. The immediate effects of insulin and fructose on the metabolism of the perfused liver. Biochem. J. 126: 295-311.

26. Tulloch, B. R., K. Dyal, and T. R. Fraser. 1972. Increased lipid synthesis by liver slice in superfusion system following raised glucose or insulin concentration. Diabetologia. 8: 267-273.

27. Heimberg, M., I. Weinstein, and M. Kahout. 1969. The effects of glucagon, dibutyryl cyclic adenosine $3^{\prime} 5^{\prime}$ mono- phosphate and concentration of free fatty acid on hepatic lipid metabolism. J. Biol. Chem. 244: 5131-5139.

28. McGarrey, J. D., P. H. Wright, and D. W. Foster. 1975 Hormonal control of ketogenesis. Rapid activation of hepatic ketogenic capacity in fed rats by anti-insulin serum and glucagon. J. Clin. Invest. 55: 1202-1209.

29. Blackard, W. G., N. C. Nelson, and S. S. Andrews. 1974 Portal and peripheral vein immunoreactive glucagon concentrations after arginine or glucose infusions. Diabetes. 23: 199-202.

30. Havel, R. J., J. P. Kane, E. O. Balasse, N. Segel, and L. V. Basso. 1970. Splanchnic metabolism of free fatty acids and production of triglycerides of very low density lipoproteins in normotriglyceridemic and hypertriglyceridemic humans. J. Clin. Invest. 49: 2017-2035.

31. Woodside, W. F., and M. Heimberg. 1972. Hepatic metabolism of free fatty acids in experimental diabetes. Isr. J. Med. Sci. 8: 309-316.

32. Schlierf, G., and E. Dorow. 1973. Diurnal patterns of triglycerides, free fatty acids, blood sugar, and insulin during carbohydrate-induction in man and their modification by nocturnal suppression of lipolysis. J. Clin. Invest. 52: 732-740.

33. Wahren, J., and P. Felig. 1976. Influence of somatostatin on carbohydrate disposal and absorption in diabetes mellitus. Lancet. 2: 1213-1216.

34. Adams, P. W., A. H. Kissebah, P. Harrigan, T. Stokes, and $V$. Wynn. 1974. The kinetics of plasma free fatty acid and triglyceride transport in patients with idiopathic hypertriglyceridemia and their relation to carbohydrate metabolism. Eur. J. Clin. Invest. 4: 149-161. 